\title{
Brain Tumor Detection based on Machine Learning Algorithms
}

\author{
Komal Sharma \\ Research Scholar \\ M.E - CSE \\ $\mathrm{CU}$, Gharuan \\ Mohali, Punjab, INDIA
}

\author{
Akwinder Kaur \\ Assistant Professor \\ M.E - CSE \\ CU, Gharuan \\ Mohali, Punjab, INDIA
}

\author{
Shruti Gujral \\ Assistant Professor \\ M.E - CSE \\ $\mathrm{CU}$, Gharuan \\ Mohali, Punjab, INDIA
}

\begin{abstract}
Automated defect detection in medical imaging has become the emergent field in several medical diagnostic applications. Automated detection of tumor in Magnetic Resonance Imaging (MRI) is very crucial as it provides information about abnormal tissues which is necessary for planning treatment. The conventional method for defect detection in magnetic resonance brain images is human inspection. This method is impractical for large amount of data. So, automated tumor detection methods are developed as it would save radiologist time. The MRI brain tumor detection is complicated task due to complexity and variance of tumors. In this paper, tumor is detected in brain MRI using machine learning algorithms. The proposed work is divided into three parts: preprocessing steps are applied on brain MRI images, texture features are extracted using Gray Level Co-occurrence Matrix (GLCM) and then classification is done using machine learning algorithm.
\end{abstract}

Keywords: Magnetic Resonance Imaging, Segmentation, Feature Extraction, Texture Features, Machine learning.

\section{INTRODUCTION}

The human body is composed of many types of cells. Each cell has a specific function. The cells in the body grow. and divide in an orderly manner and form some new cells. These new cells helps to keep the human body healthy and properly working. When some cells lose their capability to control their growth, they grow without any order. The extra cells formed form a mass of tissue which is called tumor. The tumors can be benign or malignant. Malignant tumors lead to cancer while benign tumors are not cancerous. According to a report published by central brain tumor registry of the United States (CBTRUS), approximately 39,550 people were diagnosed with benign and malignant brain tumors in 2002 . It indicates that the rate of primary brain tumor whether malignant tumor or benign tumor is 14 per 100,000 [3].

The important factor in the medical diagnosis include the medical image data obtained from various biomedical devices that uses different imaging techniques like X-ray, CT Scan, MRI. Magnetic Resonance Imaging (MRI) is a technique which depends on the measurement of magnetic field vectors that are generated after an appropriate excitation of strong magnetic fields and radio frequency pulses in the nuclei of hydrogen atoms present in the water molecules of a patient's body [5]. The MRI scan is much better than CT scan for diagnosis as it doesn't use any radiation. The radiologists can evaluate the brain using MRI. The MRI technique can determine the presence of tumor in the brain. The conventional method for tumor detection in MRI image is human inspection. This method is very time consuming. It is not appropriate for large amount of data. The MRI also contains noise caused due to operator intervention which can lead to inaccurate classification. Large volume of MRI is to analyzed, thus, automated systems are needed as it they are more cost-effective. Automated detection of tumor in MRI images is necessary as high accuracy is needed when dealing with human life.

The MR human brain images are classified by using supervised techniques like artificial neural networks, support vector machine, and unsupervised techniques like selforganization map (SOM), fuzzy c-means when combined with feature extraction techniques. Other supervised classification techniques, such as k-nearest neighbors (k-NN) also group pixels based on their similarities in each feature [19]. Classification of MR images either as normal or abnormal can be done via both supervised and unsupervised techniques.

In this paper, an efficient automated classification technique for brain MRI is proposed using machine learning algorithms. The supervised machine learning algorithm is used for classification of brain MR image.

\section{RELATED WORK}

Natarajan et al. [1] proposed brain tumor detection method for MRI brain images. The MRI brain images are first preprocessed using median filter, then segmentation of image is done using threshold segmentation and morphological operations are applied and then finally, the tumor region is obtained using image subtraction technique. This approach gives the exact shape of tumor in MRI brain image. Joshi et al. [2] proposed brain tumor detection and classification system in MR images by first extracting the tumor portion from brain image, then extracting the texture features of the detected tumor using Gray Level Co-occurrence Matrix (GLCM) and then classified using neuro-fuzzy classifier. Amin and Mageed [3] proposed neural network and segmentation base system to automatically detect the tumor in brain MRI images. The Principal Component Analysis (PCA) is used for feature extraction and then Multi-Layer Perceptron (MLP) is used classify the extracted features of MRI brain image. The average recognition rate is $88.2 \%$ and peak recognition rate is $96.7 \%$. Sapra et al. [4] proposed image segmentation technique to detect brain tumor from MRI images and then Probabilistic Neural Network (PNN) is used for automated brain tumor classification in MRI scans. PNN system proposed handle the process of brain tumor classification more accurately. Suchita and Lalit [5] proposed unsupervised neural network learning technique for classification of brain MRI images. The MRI brain images are first preprocessed which include noise filtering, edge detection, then the tumor is extracted using segmentation. The texture features are extracted using Gray-Level Co-occurrence Matrix(GLCM) and then Self-Organizing Maps (SOM) are used to classify the brain as normal or abnormal brain, that is, 
whether it contain tumor or not. Rajeshwari and Sharmila [6] proposed pre-processing techniques which are used to improve the quality of MRI image before using it into an application. The average, median and wiener filters are used for noise removal and interpolation based Discrete Wavelet Transform (DWT) technique is used for resolution enhancement. The Peak Signal to Noise Ratio (PSNR) is used for evaluation of these techniques.

George and Karnan [7] proposed MRI image enhancement technique based on Histogram Equalization and Center Weighted Median (CWM) filter as they are used to enhance the MRI image more effectively. Daljit Singh et al. in [8] proposed a hybrid technique for automatic classification of MRI images by first extracting the features using Principal Component Analysis (PCA) and Gray-Level Co-occurrence Matrix(GLCM) and then extracted features are fed as an input to Support Vector Machine(SVM) classifier which classifies the brain image as normal or abnormal. Gadpayleand and Mahajani [9] proposed brain tumour detection and classification system. The tumor is extracted using segmentation and then texture features are extracted using GLCM and finally the BPNN and KNN classifiers are used to classify the MRI brain image into normal or abnormal brain. The accuracy is $70 \%$ using KNN classifier and $72.5 \%$ by using BPNN classifier. Shasidhar et al. in [10] proposed modified Fuzzy C-Means (FCM) algorithm for MR brain tumor detection. The texture features are extracted from brain MR image and then modified FCM algorithm is used for brain tumor detection. The average speed-ups of as much as 80 times a traditional FCM algorithm is obtained using the modified FCM algorithm. The modified FCM algorithm is a fast alternative to the traditional FCM technique. Rajesh and Malar [11] proposed brain MR image classification based on Rough set theory and feed-forward neural network classifier. The features are extracted from MRI images using Rough set theory. The selected features are fed as input to Feed Forward Neural Network classifier which differentiate between normal and abnormal brain and the accuracy of about $90 \%$ is obtained.

Ramteke and Monali [14] proposed automatic classification of brain MR images in two classes Normal and Abnormal based on image features and automatic abnormality detection. The Statistical texture feature set is obtained from normal and abnormal images and then KNN classifier is used for classifying image. The KNN obtain $80 \%$ classification rate. Xuan and Liao [15] proposed statistical structure analysis based tumor segmentation technique. The intensity-based, symmetry-based and texture-based features are extracted from MR image. Then, classification technique using AdaBoost is used to classify the MR image into normal tissues and abnormal images. The average accuracy of about $96.82 \%$ is achieved. Othman et al. in [16] proposed Probabilistic neural network technique for brain tumor classification. Firstly, the features are extracted using the principal component analysis (PCA) and the classification is performed using Probabilistic Neural Network (PNN). Ibrahim et al. in [19] proposed Neural Network technique for the classification of the magnetic resonance human brain images. The features are extracted using principal Component Analysis (PCA) and then Back-Propagation Neural Network is used as a classifier to classify MRI brain images as normal or abnormal. The classification accuracy of about $96.33 \%$ is obtained. Jafari and Shafaghi [21] proposed a hybrid approach for brain tumor detection in MR images based on Support Vector Machines(SVM). The texture and intensity features are used. The accuracy of about $83.22 \%$ is achieved and is more robust.
Thus from extensive literature survey we found that most of the current brain tumor detection system uses texture, symmetry and intensity as features. Texture features are important property of brain as texture perception has a very important aspect in the human visual system of recognition and interpretation [24]. Here, we propose extracting texture features like energy, contrast, correlation, Homogenity [5]. Gray Level Co-occurrence Matrix is used for extraction of texture features.

Further we propose the use of ML algorithms to overcome the drawbacks of traditional classifiers. We investigate and compare the performance of two machine learning algorithms namely MLP and Naive Bayes in this work. Since these ML algorithms are found to perform well in most of the pattern classification tasks. Neural networks are useful as they can learn complex mappings between input and output. They are capable of solving much more complicated classification tasks. However, when certain rules cannot be modeled exactly, the concept of probability is used, which is the basis for Naive Bayes classification.

\section{PROPOSED METHOD}

As per literature survey, it was found that automated brain tumor detection is very necessary as high accuracy is needed when human life is involved. Automated detection of tumor in MR images involves feature extraction and classification using machine learning algorithm. In this paper, a system to automatically detect tumor in MR images is proposed as shown in figure 1 .

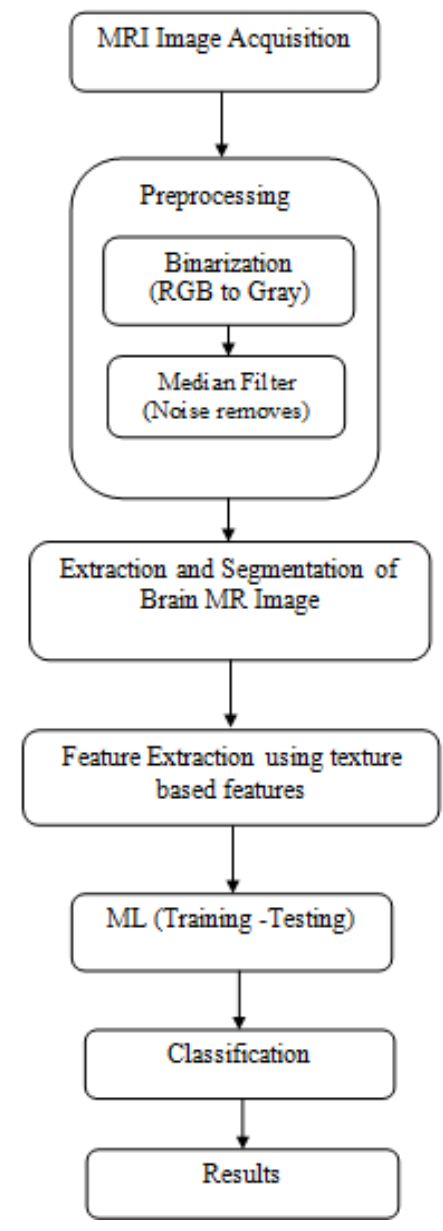

Fig 1. Proposed Method for Brain Tumor Detection in MR images 
Image Acquisition: The MRI brain images are acquired and are given as input to pre-processing stage. The sample brain MR images are shown in figure 2.
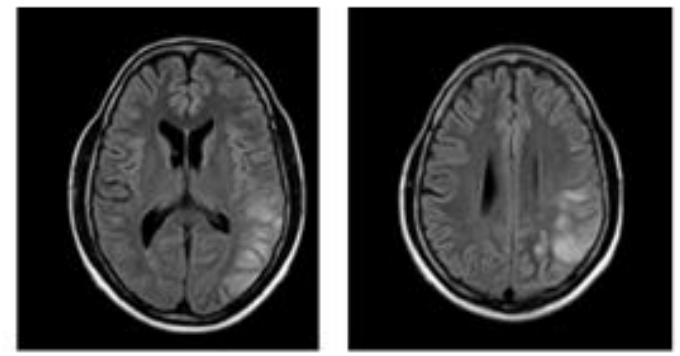

Fig 2. Samples of brain MR image

Preprocessing: Preprocessing is needed as it provides improvement in image data which enhances some of the image features which are important for further processing. The pre-processing steps that are applied to MR image are as follows :

The RGB MR image is converted to gray scale image and then median filter is applied for noise removal from brain MR images as shown in figure 3(b). The noise is to removed for further processing as high accuracy is needed.

Then edges are detected from filtered image using canny edge detection as shown in figure 3(c). The edge detected image is needed for segmentation of the image.

Then watershed segmentation is done for finding the location of the tumor in the brain image as shown in figure 3(d). Segmentation is the process of dividing an image into multiple segments. The aim of segmentation is to change representation of image into something which is more easy to analyze. The result of watershed segmentation is label image. In label image, all the different objects identified will have different pixel values, all the pixels of first object will have value 1 , all the pixels of second object will have value 2 and so on [23]. The various preprocessing operations applied on brain MR image are shown in figure 3.

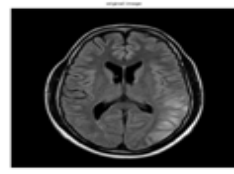

(a) Original Image

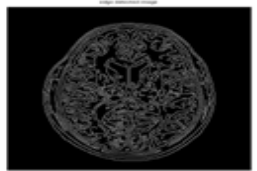

(c) Edge Detected Image

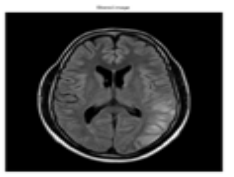

(b)Filtered Image

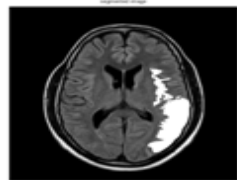

(d) Segmented Image
Fig 3(a-d). Preprocessing operations on input brain image

Feature Extraction: When input to an algorithm is very large and redundant to be processed, it is transformed into reduced representative set of features called feature vector. Transformation of input data into set of features is called feature extraction [5]. In this step, the important features needed for image classification are extracted. The segmented brain MR image is used and texture features are extracted from the segmented image which shows the texture property of the image. These features are extracted using Gray Level Co-occurrence Matrix (GLCM) as it is robust method with high performance. The GLCM texture feature extraction method is very competitive as using smaller number of gray levels shrinks the size of GLCM which reduces the computational cost of the algorithm and at the same time preserves the high classification rates. The GLCM features are used to distinguish between normal and abnormal brain.

Texture contains some important information about surface structural arrangement. The textural features based on graytone spatial dependencies have a general applicability in image classification

The GLCM texture features that are extracted are as follows:

(1) Energy : It gives a measure of textural uniformity, that is, measure of pixel pair repetitions.

$\mathrm{E}=\sum_{\mathrm{i}=0}^{\mathrm{N}_{\mathrm{g}}-1} \sum_{\mathrm{j}=0}^{\mathrm{N}_{\mathrm{g}}-1} \mathrm{p}(\mathrm{i}, \mathrm{j})^{2}$

Range $=[0,1]$

(2) Contrast : It gives a measure of intensity contrast between a pixel and its neighbor over the whole image.

Con $=\sum_{\mathrm{n}=0}^{\mathrm{N}_{\mathrm{g}}-1} \mathrm{n}^{2} \sum_{\mathrm{i}=0}^{\mathrm{N}_{\mathrm{g}}-1} \sum_{\mathrm{j}=0}^{\mathrm{N}_{\mathrm{g}}-1} \mathrm{p}(\mathrm{i}, \mathrm{j})^{2}$

Range $=[0,1]$

(3) Correlation : It gives a measure of how correlated a pixel to its neighbor over the whole image.

$\mathrm{C}=\frac{1}{\sigma^{x} \sigma^{y}} \sum_{i=0}^{N_{g}-1} \sum_{j=0}^{N_{g}-1}(i, j) p(i, j)^{2}-\mu_{x} \mu_{y}$

Range $=[-1,1]$

(4) Homogeneity : It gives a measure of closeness of distribution of elements in GLCM to GLCM diagonal.

$\mathrm{H}=\sum_{\mathrm{i}=0}^{\mathrm{N}_{\mathrm{g}}-1} \sum_{\mathrm{j}=0}^{\mathrm{N}_{\mathrm{g}}-1} \frac{\mathrm{p}(\mathrm{i}, \mathrm{j})}{(1+\bmod (\mathrm{i}, \mathrm{j}))}$

Range $=[0,1]$

Thus to classify the brain as normal or abnormal, the MRI brain images are collected, preprocessing steps and segmentation are then applied. The segmented image is used for feature extraction and texture features are extracted using GLCM. The preprocessing and feature extraction steps are done in Matlab 2012a.

(5) Classification: The Machine learning algorithms are used for classification of MR brain image either as normal or abnormal. The major aim of ML algorithms is to automatically learn and make intelligent decisions. The feature set formed by above specified method was applied to Multi-Layer Perceptron (MLP) and Naive Bayes for classification. MLP [3] is a feed forward artificial neural network model that maps sets of input data into a set of appropriate output. It is known as feed forward because it does not contain any cycles and network output depends only on the current input instance. In MLP, each node is a neuron with a nonlinear activation function. It is based on supervised learning technique. Learning take place by changing connection weights after each piece of data is processed, based on the amount of error in the target output as compared to the expected result. The goal of the learning procedure is to minimize error by improving the current values of the weight associated with each edge. Because of this backward changing process of the weights, model is named as back- propagation. 
Naive bayes is a supervised learning as well as statistical method for classification. It is simple probabilistic classifier based on Bayes theorem. It assumes that the value of a particular feature is unrelated to the presence or absence of any other feature. The prior probability and likelihood are calculated in order to calculate the posterior probability. The method of maximum posterior probability is used for parameter estimation. This method requires only a small amount of training data to estimate the parameters which are needed for classification. The time taken for training and classification is less.

\section{EXPERIMENTAL RESULTS}

The experiment was carried out on 212 brain MR images. From each image, the texture based features are extracted and weka tool is used for classification [28]. The texture based features such as energy, contrast, correlation, homogeneity are extracted using GLCM. The Multi-Layer Perceptron (MLP) and Naïve bayes with $66 \%$ percentage split is used for classification. In $66 \%$ percentage split, $66 \%$ of the instances are used for training and remaining instances are used for testing.

Table 1. Experimental result analysis

\begin{tabular}{|c|c|c|c|}
\hline $\begin{array}{c}\text { ML } \\
\text { Algorithm }\end{array}$ & $\begin{array}{c}\text { Total } \\
\text { samples }\end{array}$ & $\begin{array}{c}\text { Model Build } \\
\text { Time }\end{array}$ & $\begin{array}{c}\text { Classification } \\
\text { Rate }(\%)\end{array}$ \\
\hline MLP & 210 & 61.82 & 98.6 \\
\hline $\begin{array}{c}\text { Naive } \\
\text { bayes }\end{array}$ & 210 & 0.02 & 97.6 \\
\hline
\end{tabular}

From the Table 1, we can find the classification rate of brain MR images using MLP and Naive bayes. The accuracy of about $98.6 \%$ and $91.6 \%$ is obtained respectively.

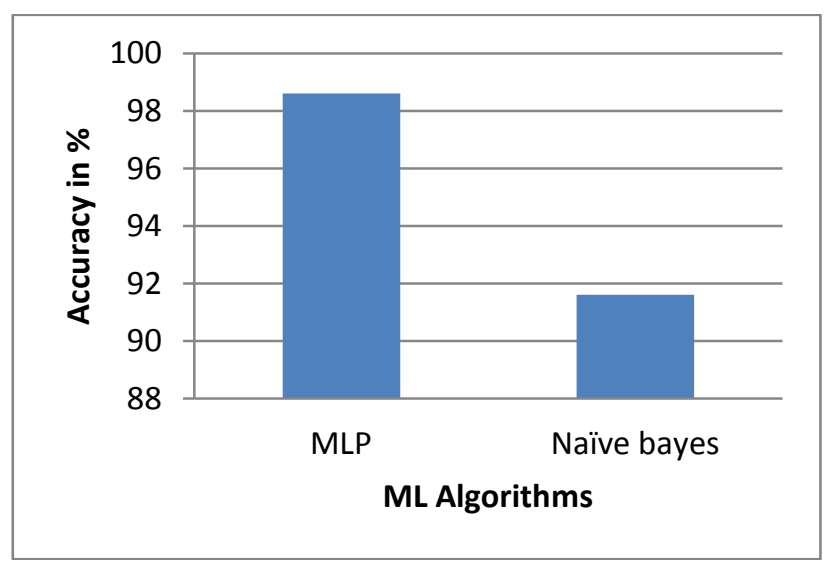

Fig 4. Graphical representation of accuracy

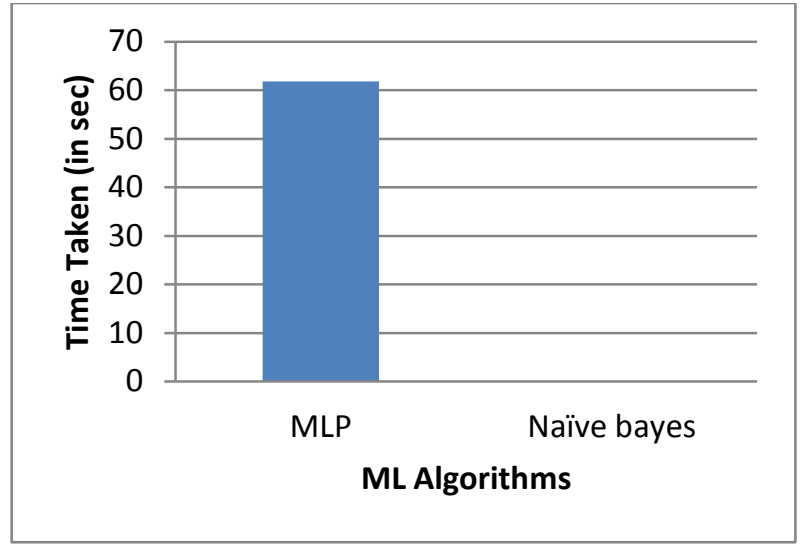

Fig 5. Graphical representation of time taken

The MLP gives more accuracy and takes more time to build the model while the time taken by Naïve Bayes is less and is less accurate. Due to diverse appearances and complexity of tumors, the proposed method gives the satisfied accuracy. The high accuracy is desirable as human life is involved.

\section{CONCLUSION}

This paper proposes a work on brain tumor detection system based on machine learning algorithms. The texture based features are extracted using Gray Level Co-occurrence Matrix (GLCM). The texture features of the image considered in this proposed work include energy, contrast, correlation, homogeneity. For the classification purpose, Multi-Layer Perceptron and Naïve bayes machine learning algorithm is used and the maximum accuracy $98.6 \%$ and $91.6 \%$ is achieved by considering 212 samples of brain MR images. This accuracy can probably be increased by considering a large data set and extracting intensity based features in addition to the texture based features.

\section{REFERENCES}

[1] Natarajan P, Krishnan.N, Natasha Sandeep Kenkre, Shraiya Nancy, Bhuvanesh Pratap Singh, "Tumor Detection using threshold operation in MRI Brain Images" , IEEE International Conference on Computational Intelligence and Computing Research, 2012.

[2] Dipali M. Joshi, N. K. Rana, V. M. Misra, " Classification of Brain Cancer Using Artificial Neural Network" , IEEE International Conference on Electronic Computer Technology ,ICECT ,2010.

[3] Safaa E.Amin, M.A. Mageed," Brain Tumor Diagnosis Systems Based on Artificial Neural Networks and Segmentation Using MRI" , IEEE International Conference on Informatics and Systems, INFOS 2012.

[4] Pankaj Sapra, Rupinderpal Singh, Shivani Khurana, "Brain Tumor Detection Using Neural Network" , International Journal of Science and Modern Engineering, IJISME ,ISSN: 2319-6386, Volume-1, Issue-9, August 2013.

[5] Suchita Goswami, Lalit Kumar P. Bhaiya, " Brain Tumor Detection Using Unsupervised Learning based Neural Network" , IEEE International Conference on 
Communication Systems and Network Technologies,2013.

[6] S. Rajeshwari, T. Sree Sharmila, "Efficient Quality Analysis of MRI Image Using Preprocessing Techniques", IEEE Conference on Information and Communication Technologies, ICT 2013.

[7] E. Ben George, M.Karnan, "MRI Brain Image Enhancement Using Filtering Techniques", International Journal of Computer Science \& Engineering Technology, IJCSET, 2012.

[8] Daljit Singh, Kamaljeet Kaur, "Classification of Abnormalities in Brain MRI Images Using GLCM, PCA and SVM" , International Journal of Engineering and Advanced Technology (IJEAT) ISSN: 2249 - 8958, Volume-1, Issue-6, August 2012.

[9] Prachi Gadpayleand, P.S. Mahajani, "Detection and Classification of Brain Tumor in MRI Images ", International Journal of Emerging Trends in Electrical and Electronics, IJETEE - ISSN: 2320-9569, Vol. 5, Issue. 1, July-2013.

[10] M. Shasidhar, V.Sudheer Raja, B. Vijay Kumar, "MRI Brain Image Segmentation Using Modified Fuzzy CMeans Clustering Algorithm" ,IEEE International Conference on Communication Systems and Network Technologies, 2011.

[11] T. Rajesh, R. Suja Mani Malar," Rough Set Theory and Feed Forward Neural Network Based Brain Tumor Detection in Magnetic Resonance Images" ,IEEE International on Advanced Nanomaterials \& Emerging Engineering Technologies, 2013.

[12] Komal Sharma, Navneet Kaur, " Comparative Analysis of Various Edge Detection Techniques" , International Journal of Advanced Research in Computer Science and Software Engineering, IJARCSSE, ISSN: 2277 128X, Volume 3, Issue 12, December 2013.

[13] J. Selvakumar, A. Lakshmi, T. Arivoli, " Brain Tumor Segmentation and Its Area Calculation in Brain MR Images using K-Mean Clustering and Fuzzy C-Mean Algorithm" , IEEE-International Conference On Advances In Engineering, Science And Management, ICAESM, 2012.

[14] R. J. Ramteke1, Khachane Monali Y., " Automatic Medical Image Classification and Abnormality Detection Using K-Nearest Neighbour" , International Journal of Advanced Computer Research,Volume-2 Number-4 Issue-6 December-2012.

[15] Xiao Xuan, Qingmin Liao, Statistical Structure Analysis in MRI Brain Tumor Segmentation" ,IEEE International Conference on Image and Graphics, 2007.

[16] Mohd Fauzi Othman, Mohd Ariffanan, Mohd Basri, " Probabilistic Neural Network for Brain Tumor Classification" ,IEEE International Conference on Intelligent Systems, Modelling and Simulation,2011.
[17] Shweta Jain, "Brain Cancer Classification Using GLCM Based Feature Extraction in Artificial Neural Network" , International Journal of Computer Science \& Engineering Technology ,IJCSET, ISSN : 2229-3345 Vol. 4 No. 07 Jul 2013.

[18] Dina Aboul Dahab, Samy S. A. Ghoniemy, Gamal M. Selim, "Automated Brain Tumor Detection and Identification using Image Processing and Probabilistic Neural Network Techniques" ,International Journal of Image Processing and Visual Communication, ISSN 2319-1724 : Volume (Online) 1, Issue 2, October 2012.

[19] Walaa Hussein Ibrahim, Ahmed Abdel Rhman Ahmed Osman, Yusra Ibrahim Mohamed, "MRI Brain Image Classification Using Neural Networks" ,IEEE International Conference On Computing, Electrical and Electronics Engineering, ICCEEE,2013.

[20] Noramalina Abdullah, Lee Wee Chuen, Umi Kalthum Ngah Khairul Azman Ahmad, "Improvement of MRI Brain Classification using Principal Component Analysis" , IEEE International Conference on Control System, Computing and Engineering, 2011.

[21] Mehdi Jafari, Reza Shafaghi, "A Hybrid Approach for Automatic Tumor Detection of Brain MRI using Support Vector Machine and Genetic Algorithm", Global Journal of Science Engineering and Technology, Issue-3, 2012.

[22] V. Salai Selvam and S. Shenbagadevi, "Brain Tumor Detection using Scalp EEG with Modified Wavelet-ICA and Multi Layer Feed Forward Neural Network" , Annual International Conference of the IEEE EMBS Boston, Massachusetts USA, August 30 - September 3 , 2011.

[23] Amir Shahzad, Muhammad Sharif, Mudassar Raza, Khalid Hussai, " Enhanced Watershed Image Processing Segmentation " , Journal of Information \& Communication Technology, Vol. 2, No. 1, 2009.

[24] Neelam Marshkole, Bikesh Kumar Singh, A.S Thoke, "Texture and Shape based Classification of Brain Tumors using Linear Vector Quantization" , International Journal of Computer Applications (0975 - 8887) Volume 30-No.11, September 2011.

[25] MATLAB Central, URL: http://www.mathworks.in/discovery/imagesegmentation. html.

[26] MATLAB Central, URL: http://www.mathworks.in/matlabcentral/fileexchange/22 187 -glcm texture-features.

[27] MATLAB Central, URL: http://www.mathworks.in/help/images/analyzing-thetexture-of-an-image.html.

[28] WEKA 3: Data Mining With Open Source Machine Learning Software in JAVA, URL: http://www.cs.waikato.ac.nz/ml/weka/. 